\title{
Plumbagin protects liver against fulminant hepatic failure and chronic liver fibrosis via inhibiting inflammation and collagen production
}

\author{
Huafeng Wang ${ }^{1,2,3, *}$, Huan Zhang ${ }^{1,4, *}$, Yuqing Zhang ${ }^{5, *}$, Dan Wang ${ }^{1, *}$, Xixi Cheng ${ }^{1}$, \\ Fengrui Yang ${ }^{1,2}$, Qi Zhang ${ }^{1}$, Zhenyi Xue ${ }^{1,2}$, Yan Li ${ }^{1,2}$, Lijuan Zhang ${ }^{1,2}$, Luhong Yang ${ }^{3}$, \\ Guolin Miao ${ }^{1,2}$, Daiqing Lí, Zhiyu Guan7, Yurong $\mathrm{Da}^{1,2}$, Zhi Yao' ${ }^{2}$, Fei Gao ${ }^{8}$, Liang \\ Qiao9, Li Kong ${ }^{10}$, Rongxin Zhang ${ }^{1,2}$ \\ ${ }^{1}$ Laboratory of Immunology and Inflammation, Department of Immunology and Research Center of Basic Medical Sciences, \\ Tianjin Medical University, Tianjin, China \\ ${ }^{2}$ Department of Immunology, Tianjin Key Laboratory of Cellular and Molecular Immunology, Key Laboratory of Immune \\ Microenvironment and Diseases, Ministry of Education of China, Tianjin Medical University, Tianjin, China \\ ${ }^{3}$ School of Life Science, Shanxi Normal University, Linfen, China \\ ${ }^{4}$ Clinical Laboratory, Tianjin Academy of Traditional Chinese Medicine Affiliated Hospital, Tianjin, China \\ ${ }^{5}$ Department of Hepatobiliary and Pancreatic Surgery, Tianjin Nankai Hospital, Nankai Clinical School of Medicine, Tianjin \\ Medical University, Tianjin, China \\ ${ }^{6}$ Key Laboratory of Hormones and Development (Ministry of Health), Metabolic Diseases Hospital and Tianjin Institute of \\ Endocrinology, Tianjin Medical University, Tianjin, China \\ ${ }^{7}$ Department of Pathogenic Biology, Weifang Medical University, Shandong, China \\ ${ }^{8}$ State Key Laboratory of Reproductive Biology, Institute of Zoology, Chinese Academy of Sciences, Beijing, China \\ ${ }^{9}$ Storr Liver Unit, Westmead Millennium Institute, The Western Clinical School of the University of Sydney, Westmead, NSW, \\ Australia \\ ${ }^{10}$ Department of Histology and Embryology, Dalian Medical University, Dalian, China \\ *These authors have contributed equally to this work \\ Correspondence to: Rongxin Zhang, email: rxzhang@tmu.edu.cn, rongxinz@yahoo.com \\ Li Kong, email: kongli@dmu.edu.cn
}

Keywords: plumbagin, fulminant hepatic failure, liver fibrosis, hepatic stellate cell, inflammation

Received: August 08, 2016

Accepted: October 04, 2016

Published: October 14, 2016

\section{ABSTRACT}

Plumbagin is a quinonoid constituent extracted from Plumbago genus, and it exhibits diverse pharmacological effects. This study thoroughly investigated the effects of plumbagin on thioacetamide-induced acute and chronic liver injury. Results shown that plumbagin increased survival rate, reduced liver congestion and inflammation, and decreased macrophages and neutrophils in the fulminant hepatic failure model, and remarkably diminished liver fibrosis and inflammation in the chronic liver injury model. Furthermore, plumbagin significantly suppress the HSCs/ myofibroblasts activation by reduced expression of markers a-SMA and COL-1/3, and reduced macrophage in liver. In the in vitro study, plumbagin induced apoptosis and suppressed the proliferation of LX-2 cells (human HSCs). Plumbagin treatment increased AMPK phosphorylation and attenuated NF-kB, STAT3, and Akt/mTOR signals in LX-2 cells, while SMAD2 phosphorylation was not changed. Noticeably, plumbagin promoted AMPK binding to p300 which is a cofactor of SMAD complex, this may further competitively decreases the p300/SMAD complex initiated transcription of COL-1/3 and a-SMA. Additionally, plumbagin hampered inflammation related NF-kB signal in RAW 264.7 cells. In conclusion, these findings indicate that plumbagin may be a powerful drug candidate to protect the liver from acute and chronic damage by inhibiting inflammation and collagen production. 


\section{INTRODUCTION}

The liver is the largest solid organ in the body, and it plays a pivotal role in metabolism and exhibits an alexipharmic function in the body [1]. Liver damage is a major disease that seriously threatens human health worldwide, especially in Asian countries. Fulminant hepatic failure (FHF) is caused by a variety of factors, such as viral infection, drug damage and food poisoning [2]. FHF often presents with extensive hepatocyte necrosis and severe liver function abnormalities. FHF is a concern because of its acute onset, rapid progression and high fatality rate. Liver fibrosis is a common chronic liver disease that is caused by variety of pathogenic factors [3]. Liver fibrosis is not an independent disease, and it is always accompanied with chronic hepatitis.

The root of Plumbago zeylanica $L$ has been used for centuries in traditional Indian and Chinese medicine for the treatment of various ailments. Plumbagin (5-hydroxy-2methyl-1,4 naphthoquinone) is a quinonoid constituent that is found in the roots of medicinal herbs of the Plumbago genus [4], and it exhibits diverse pharmacological effects. Hyperlipidemic rabbits that received plumbagin exhibited a definite regression of atheroma and reduced accumulation of cholesterol and triglycerides in the liver and aorta [5]. Plumbagin induces tumor regression in 3-methyl-4-dimethyl aminoazobenzene -induced hepatoma in Wistar male rats when administered orally at a dose of $4 \mathrm{mg} / \mathrm{kg}$ body weight [6]. Plumbagin also inhibits azoxymethane-induced intestinal carcinogenesis in rats [7]. Plumbagin treatment exerted negative effects on the invasion, migration and adhesion of HepG2 cells in vitro by decreasing MMP-2 and u-PA expression and enhancing TIMP-2 and PAI-1 expression [8]. Previous research reported the protective effects of plumbagin on liver disease, but these studies focused more on glucose metabolism and hepatocarcinogenesis $[6,8]$. Therefore, knowledge of the effects of plumbagin on FHF and liver fibrosis is very poor.

Recently, we have found that several herbal compounds derived from medicinal plants exerts potent the anti-inflammatory and anti-tumor effects [9-12], the herbal compound plumbagin exerts anti-inflammatory effects on central nervous system inflammation in experimental autoimmune encephalomyelitis [13], these suggests that plumbagin may also play anti-inflammatory role in other inflammatory diseases such as inflammatory liver injury. Thioacetamide (TAA)-induced liver injury model is widely used to study the acute-toxic liver injury and chronic liver inflammation and fibrosis. We have reported that adiponectin-derived active peptide ADP355 exerts anti-inflammatory and anti-fibrotic activities in TAA-induced liver injury [14], and mesenchymal stem cells (MSCs) secreted molecules predominantly ameliorate TAA-induced fulminant hepatic failure [15]. In this study we performed a deeper and extensive investigation of the effects of plumbagin on acute and chronic liver injury. We constructed the animal models of TAA-induced liver damage $[14,15]$ and examined the protective effects of plumbagin on acute and chronic liver injury in mice using inflammatory infiltration, hepatocytes protection, and fibrogenesis. We also investigated the possible molecular mechanism involved in plumbagin protective effects using LX-2 cells and RAW264.7 cells in vitro.

\section{RESULTS}

\section{Plumbagin improved survival rate and reduced the risk of thrombosis in the FHF model}

To verify the protective effect of plumbagin on acute liver injury, plumbagin ( $2 \mu \mathrm{g} / \mathrm{g}$ bw, once daily) [16] was administered intragastrically to ICR female mice before TAA injection until the end of the experiment and 2 days later FHF model was induced using a single intraperitoneal injection of TAA $(300 \mu \mathrm{g} / \mathrm{g} \mathrm{bw})[14,15]$. Survival rates after TAA administration in TAA mice $(\mathrm{n}=16)$ were $81.3 \%(12 \mathrm{~h}), 56.3 \%(24 \mathrm{~h})$, and $37.5 \%$ (48 $\mathrm{h}$ ), and remained $37.5 \%$ to $96 \mathrm{~h}$ later. Survival rates in PL+TAA mice ( $\mathrm{n}=14)$ were $85.7 \%(24 \mathrm{~h})$ and remained $85.7 \%$ to $96 \mathrm{~h}$ later. There was a significant difference in survival rates after TAA administration between TAA and PL+TAA mice $(\mathrm{P}=0.013$ using the log-rank test, $\mathrm{P}=$ 0.017 using Wilcoxon's test) (Figure 1A). ALP and ALT levels, which are markers of liver function, were higher in TAA mice $96 \mathrm{~h}$ after TAA administration, but these levels were lower with plumbagin treatment (Figure 1B). Livers from the TAA group were obviously congested after TAA injection, but this congestion was remarkably attenuated in PL+TAA mice (Figure 1C).

\section{Plumbagin inhibited the inflammatoryreaction and depressed macrophage and neutrophil in the FHF model}

Histological analyses of livers using HE staining revealed massive congestion around the central veins in TAA mice, and this congestion was attenuated in PL+TAA mice $24 \mathrm{~h}$ after TAA injection. Some inflammatory responses remained in TAA mice, but the primary damaged areas were repaired in PL+TAA mice $96 \mathrm{~h}$ after TAA administration (Figure 2A). Histology analysis by the Ishak's scoring system $[14,15]$ showed that necroinflammation was significantly reduced with plumbagin (Figure 2B). We next addressed whether macrophages increased in livers. The expression of F4/80 protein (macrophage marker) increased sharply in the TAA group $48 \mathrm{~h}$ after TAA administration. Differences between the TAA and PL+TAA groups were prominent (Figure 2C, 2D). It was demonstrated that hepatic infiltration of neutrophils (indicated as myeloperoxidase (MPO), Figure 2E) and hepatic production of monocyte 
chemotactic protein 1(MCP-1) (Figure 2F) were reduced with plumbagin treatment in TAA-induced fulminant liver failure model.

\section{Plumbagin reversed TAA-induced liver fibrosis, inflammation and liver function abnormalities in chronic liver model}

To further clarify the protective effect of plumbagin on chronic liver damage, An experimental hepatic fibrosis model was established with TAA $(200 \mu \mathrm{g} / \mathrm{g}$ bw ip, 3 times weekly) injection intraperitoneally into mice for six weeks. Plumbagin $(2 \mu \mathrm{g} / \mathrm{g} / \mathrm{day}$ bw ig) was administered after 6 TAA administrations until end of experiment. Liver fibrogenesis and inflammation were remarkably hampered by plumbagin treatment (Figure 3A, 3B). Liver fibrosis stage and necroinflammatory scores in the Ishak system demonstrated that the differences between TAA mice and $\mathrm{TAA}+\mathrm{PL}$ mice were statistically significant (Figure 3A,
3B). The expression of the macrophage marker F4/80 was suppressed by plumbagin in TAA+PL mice (Figure $3 C)$. The liver function abnormalities in TAA mice, such as increased serum ALP/ALT levels, were reversed by plumbagin in TAA+PL mice. Glycogen metabolism is an important function of the liver $[14,15]$. A cytoplasm filled with red glycogen particles was observed using periodic acid Schiff (PAS) staining. Glycogen massively disappeared due to reduced hepatocytes in livers from TAA mice, and many of these cells reappeared in livers from $\mathrm{TAA}+\mathrm{PL}$ mice (Figure 3E).

\section{Plumbagin therapy diminished chronic liver fibrosis by targeting HSCs/myofibroblasts}

HSCs/myofibroblasts stimulate the process of liver fibrosis $[14,17]$. As expected, strong positive staining for $\alpha$-SMA, which is a marker of HSCs/myofibroblasts [14], was detected in TAA mice, and this staining was greatly
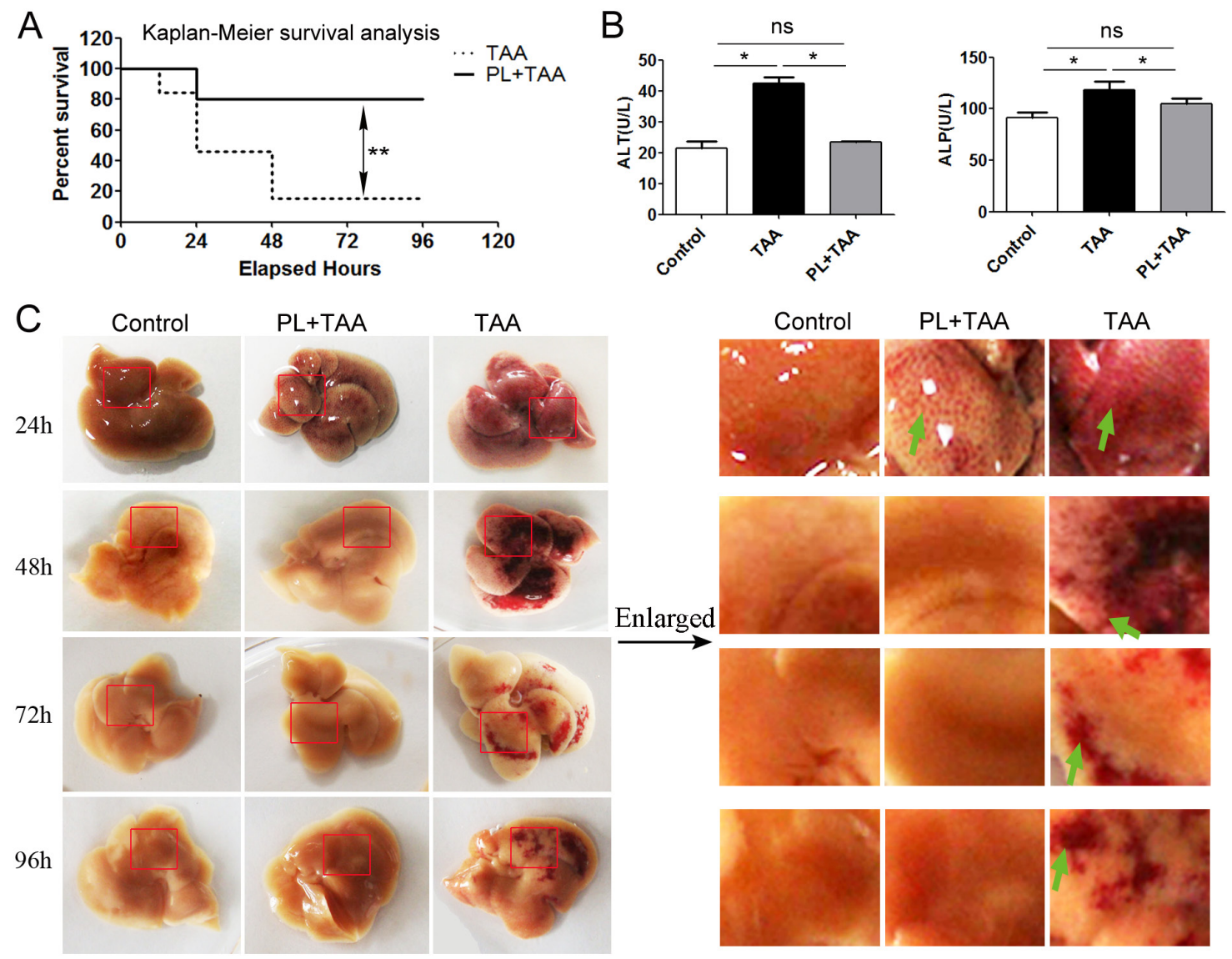

Figure 1: Plumbagin protected mice from fulminant hepatic failure. A. Survival rates of PL+TAA and TAA mice. Acute liver injury was established using a single injection of $4 \% \mathrm{TAA}(300 \mu \mathrm{g} / \mathrm{g}$ bw ip). The presence of plumbagin $(2 \mu \mathrm{g} / \mathrm{g}$ bw ig) effectively reduced TAA-induced death. B. The level of ALP/ALT $96 \mathrm{~h}$ after intraperitoneal TAA infusions. C. Gross histology of livers. Acute liver injury was induced, and mice were sacrificed at the progressive daily points. Left: Gross liver histology. Right: Enlargement of liver tissues (in red circle of the left). The presence of plumbagin significantly diminished the congestion (arrows denote) due to TAA-induced hemorrhage. Representative images are shown for all panels. Mean $\pm \mathrm{SD}$, ns (not significant), ${ }^{*} \mathrm{p}<0.05,{ }^{* *} \mathrm{p}<0.01$ were calculated using two-tailed Student's t test (T TEST). 
reduced in TAA $+\mathrm{PL}$ mice (Figure $4 \mathrm{~A}$ ). The deposition of liver ECM indicated that the production of type $1 / 3$ collagens was markedly suppressed in TAA+PL mice compared to TAA mice (Figure 4B, $4 \mathrm{C}$ ). TGF- $\beta 1$ signaling is required for $\mathrm{HSC} /$ myofibroblasts activation [14, 18]. Immunostaining revealed that plumbagin treatment decreased the expression of TGF- $\beta 1$ proteins in TAAinduced chronic liver fibrosis (Figure 4D).

\section{Plumbagin enhanced liver regeneration and reduced liver apoptosis in the TAA-induced chronic liver fibrosis model}

We hypothesized that the restoration of liver function in TAA+PL mice was related to liver regeneration. Liver regeneration marked by Ki67 occurred in response to damage in TAA and TAA+PL mice, but liver regeneration in TAA+PL mice was higher (Figure 5A). Liver apoptosis levels were detected using cleaved caspase-3 staining. Plumbagin therapy diminished liver apoptosis in the TAA+PL group (Figure 5B). It was suggested that PL exerts its beneficial effect on liver regeneration predominantly through enhancing the proliferation of hepatocytes.

\section{Plumbagin suppressed TGF- $\beta 1$ signal by the activation of AMPK in human hepatic stellate cells (LX-2) in vitro}

We addressed whether plumbagin suppressed HSCs in vitro. The inactivation of HSCs is associated AMPK [14, 19]. Plumbagin induced AMPK phosphorylation in LX-2 cells, and peak phosphorylation occurred at $1 \mathrm{~h}$ (Figure 6A). pSMAD2 levels were not significantly different between groups with or without plumbagin (Figure 6B). AMPK is a powerful competitor of SMAD complex for the interaction with p300 [20] and plumbagin-stimulated AMPK highly interacted with p300 (Figure 6C and Figure 7 ). The expression of TGF- $\beta 1$ signal downstream genes (also liver fibrosis-associated genes) such as COL1,
A
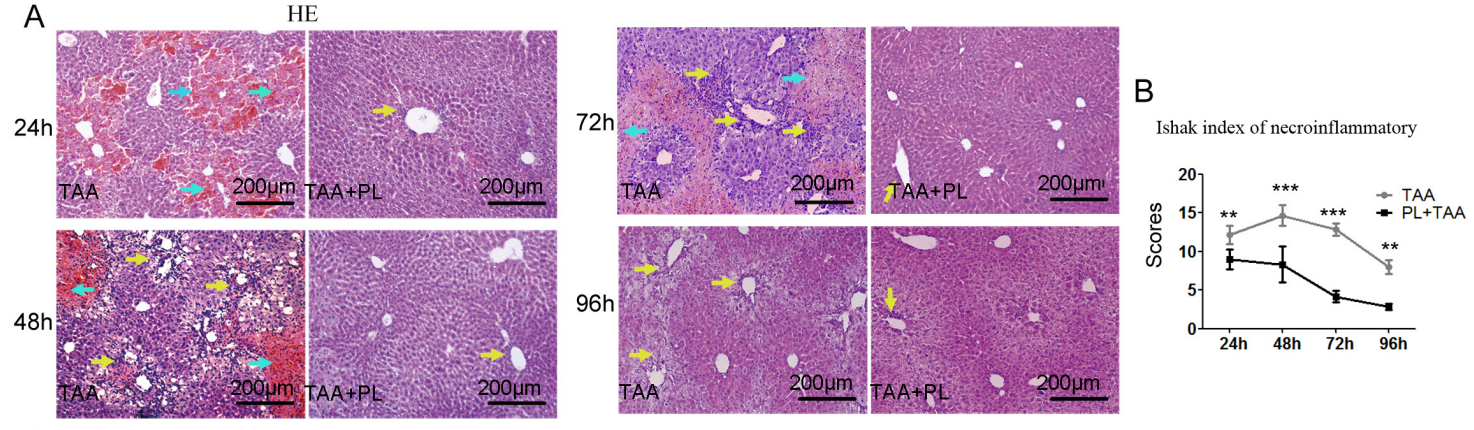

c
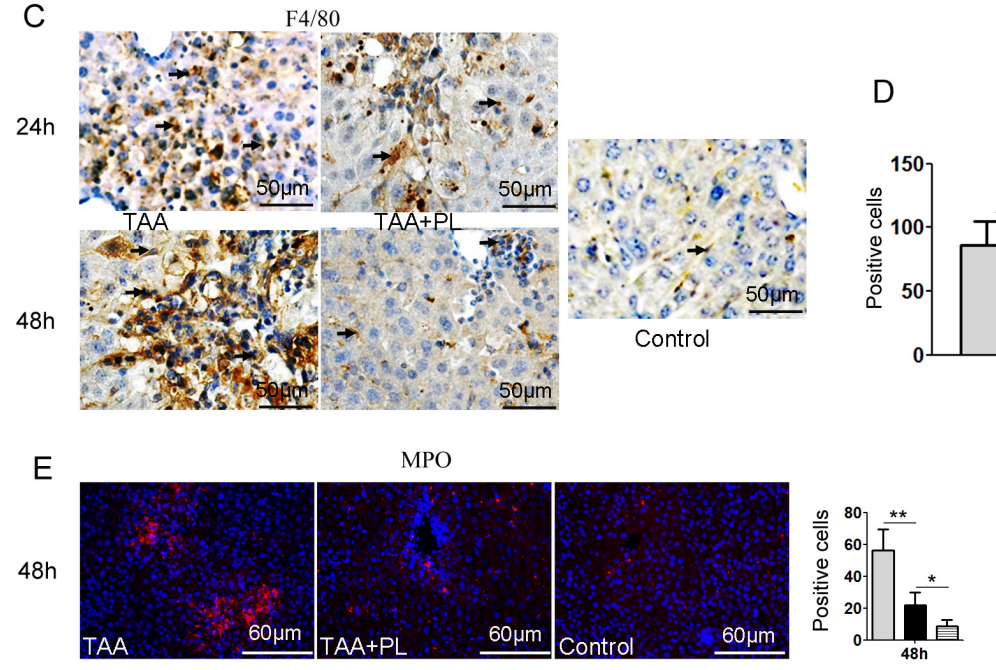

D
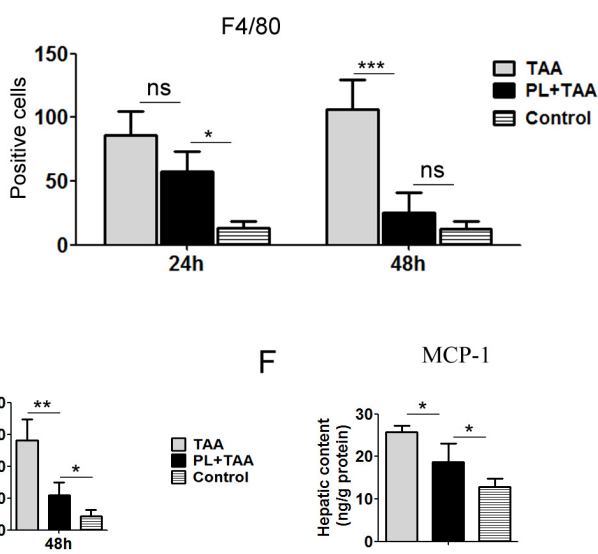

Figure 2: Plumbagin decreased congestion and inflammatory cell infiltration and macrophage recruitment in the FHF model. A. Congestion (cyan arrows) and inflammatory cell infiltration (yellow arrows) was assayed using HE staining. B. Necroinflammatory scores were determined using the Ishak classification. C. Immunostaining of F4/80 (black arrows). D. Positive cells of F4/80 were assessed by manual method. E. Immunostaining of MPO. Left panel, pictures; right panel, quantification of left images. F. Hepatic content of MCP-1. Representative images are shown for all panels. ns (not significant), ${ }^{*} \mathrm{p}<0.05,{ }^{*} \mathrm{p}<0.01 ; * * * \mathrm{p}<0.001$. Data are expressed as means $\pm \mathrm{SD}$. Statistical analyses were performed using Student's t test. 
COL3 and $\alpha$-SMA in LX-2 cells was greatly reduced by plumbagin (Figure 6D).

It was also observed that plumbagin sharply inhibited other signals, such as pSTAT3, NF-кB/p65, and Akt/mTOR in LX-2 cells (Figure 6B), which all are involved in inflammation and liver fibrosis [21-25]. In addition, NF- $\kappa$ B/p65 activation in RAW 264.7 cells was decreased by plumbagin (Supplementary Figure 1).

\section{DISCUSSION}

Plumbago genus has been shown diverse pharmacological effects on various ailments, and its extractive, known as plumbagin [4-8]. Acute liver failure (also referred to as fulminant hepatic failure) is a rare disorder that is associated with very high mortality [2]. Chronic liver failure, featured as fibrosis, is common over the world [3]. Owing to overcapacity of liver function, people with chronic liver injury are often unconscious at beginning, and conscious until to advanced stage, featured as liver cirrhosis or developed into the hepatoma [3]. Whether liver failure is acute or chronic, orthotopic liver transplantation is the current gold standard of care, but its application is limited because of organ donor shortage, financial considerations, and the requirement for lifelong immunosuppression [2, 3]. In the present animal model of FHF, we found that plumbagin treatment protected the mice against substantial lethality, and significantly decreased serum ALT and ALP levels in the TAA-induced acute inflammatory response. Plumbagin treatment simultaneously improved histological changes in hepatic lobules, such as hemorrhaging and necrosis. In the experiments of chronic liver injury, we demonstrated that plumbagin treatment remarkably suppressed liver fibrogenesis and inflammation and promoted the restoration of liver function indicated with serum ALP/ ALT levels and glycogen metabolism, which related with liver regeneration and apoptosis $[14,15]$. Stimulation of
A
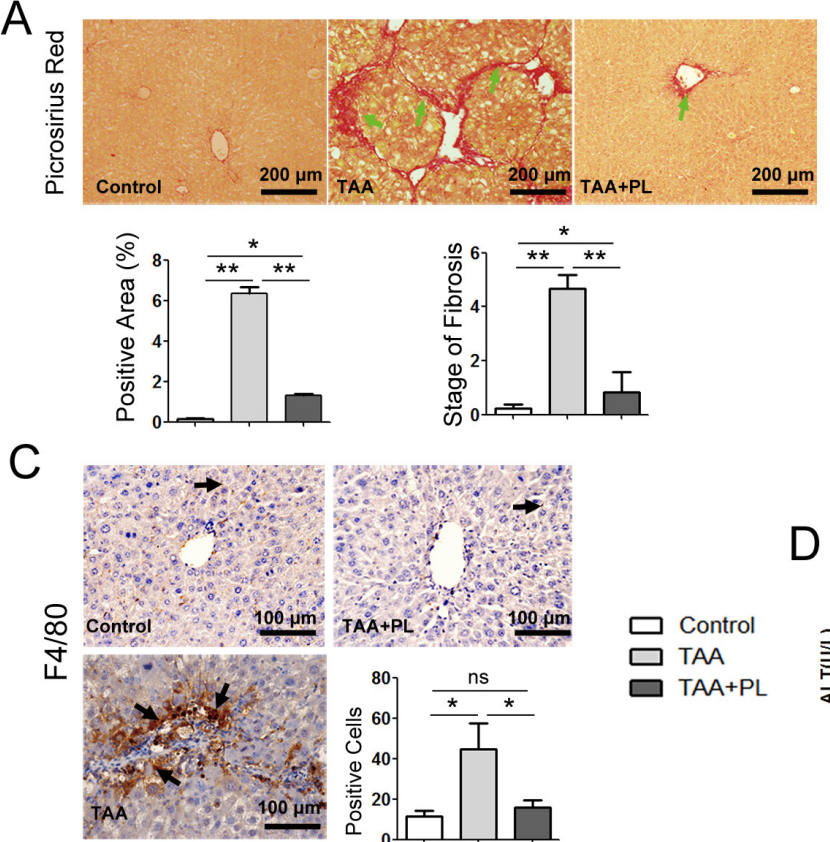

$E$

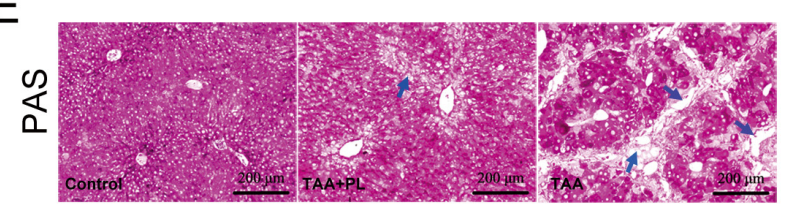

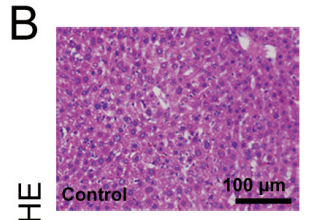
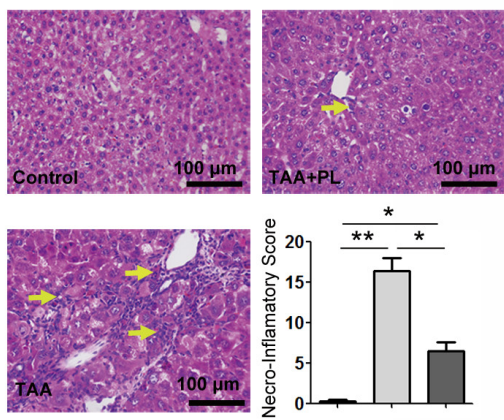
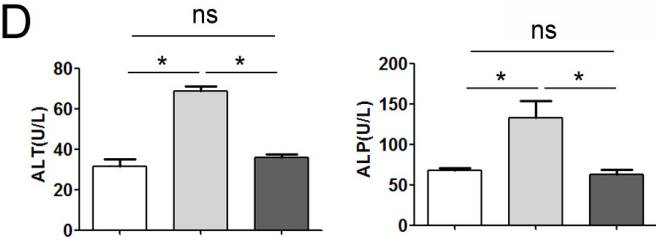

Figure 3: Plumbagin suppressed TAA-induced collagen deposition, inflammatory cell infiltration, and liver function abnormalities in a chronic liver damage model. A. Fibrosis (green arrows) was determined using sirius red staining. B. Infiltration of immune cells (yellow arrows) was shown using HE. C. F4/80 protein (black arrows) was measured using immunohistochemical staining (brown, HRP-conjugated and developed using DAB). Sections were counterstained with hematoxylin (dark blue). D. ALP and ALT levels. E. Periodic acid Schiff staining for hepatocellular glycogen storage (glycogen in red). Patches of periodic acid Schiff negative, nonfunctional hepatocytes were shown by blue arrows. Histopathological evaluations were conducted using Ishak's scoring system and Image-Pro Plus 6 Windows Software. Representative images are shown for all panels. Data are expressed as means $\pm \mathrm{SD}$. ns (not significant), ${ }^{*} \mathrm{p}<0.05 ; * * \mathrm{p}<$ 0.01. $\mathrm{p}$ values were calculated using two-sided Student's t tests. 
hepatocytes proliferation is tightly correlated with the restoration of liver structure and function. Our results also showed that expression of Ki67 (marked as liver regeneration) increased while cleaved caspase-3 (marked as liver apoptosis) decreased in livers with plumbagin treatment. Taken together, it was suggested that plumbagin can exert pharmacological effects on acute and chronic liver injury. However, in the present study, action of plumbagin was prophylactic in FHF model and detail therapeutic effects will be needed to examine in the future.

The massive infiltration of inflammatory cells is generally accompanied with the recruitment of macrophages in acute and chronic liver damage $[15,26]$. Macrophages play a crucial role in the regulation of liver inflammation [26]. The number of macrophages rises significantly in damaged livers, and these cells primarily distribute over regions of injury and fibrosis [14, 15, $27,28]$. In addition, neutrophils are also correlated with initiation of liver injury and inflammatory responses in experimental model of acute liver failure [29, 30]. Our study demonstrated a significant increase in macrophage recruitment and other inflammatory cell infiltration such as neutrophil, and related inflammatory cytokine MCP1 , as a result of liver damage, and plumbagin treatment strikingly decreased these factors in acute and chronic liver injury models. The pro-inflammatory function of hepatic macrophages was associated with the NF- $\mathrm{BB}$ signaling pathway in an alcohol-induced inflammation model [31]. Plumbagin suppressed the NF- $\kappa B$ signaling pathway in RAW264.7 cells in vitro in our study, which
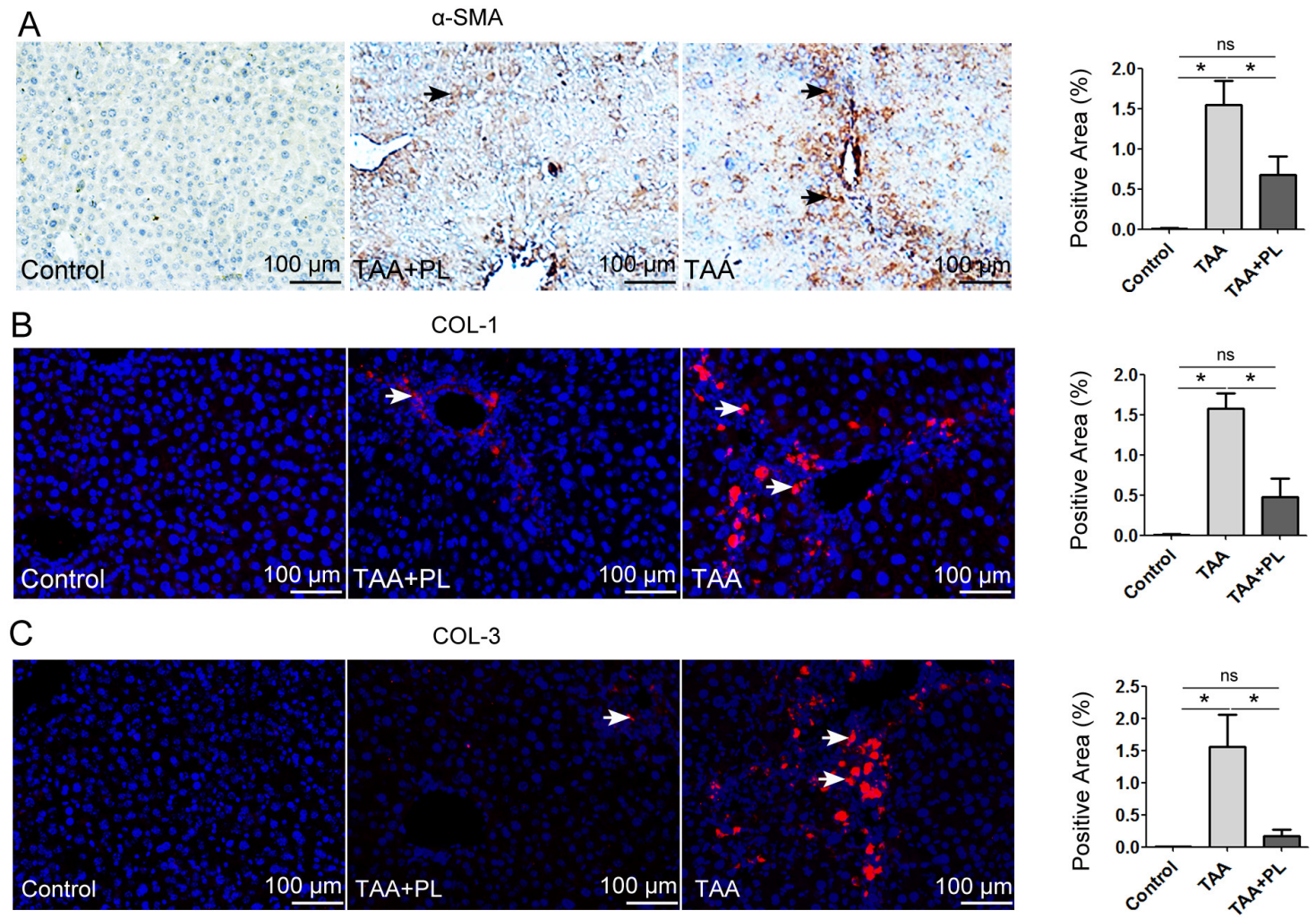

D

TGF- $\beta 1$
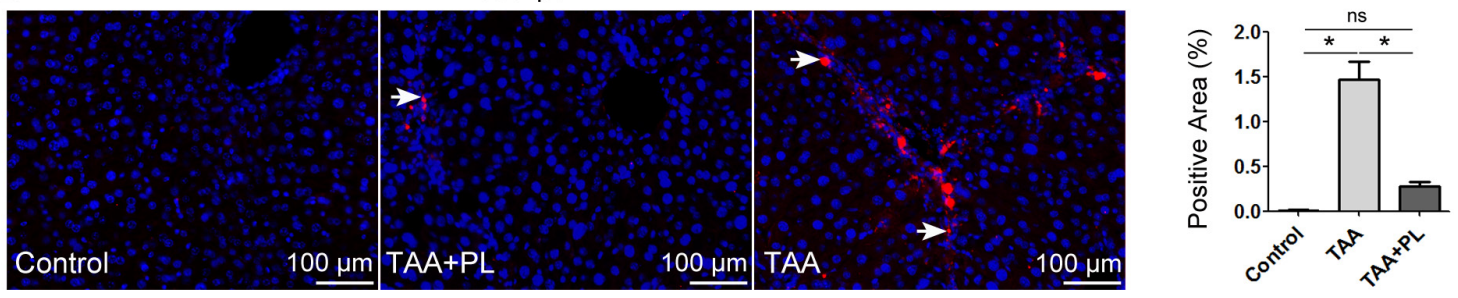

Figure 4: Immunofluorescence assays of HSCs/myofibroblasts. A. $\alpha$-SMA, a marker of activated HSCs/myofibroblasts; B. COL1 and C. COL-3, markers of ECM produced by HSCs/myofibroblasts; D. TGF- $\beta 1$. The TGF- $\beta 1$ signal is involved in HSCs/myofibroblasts. HRP-(brown) or Alexa 555 (red)-conjugated secondary antibodies were used. The sections were counterstained with hematoxylin (dark blue) or DAPI (blue). Representative images are shown for all panels. The column charts given in figure are means \pm SD values from three separate experiments. ${ }^{*} \mathrm{p}<0.05 ;{ }^{*} \mathrm{p}<0.01$. Statistical analyses were performed using Student's t test. 

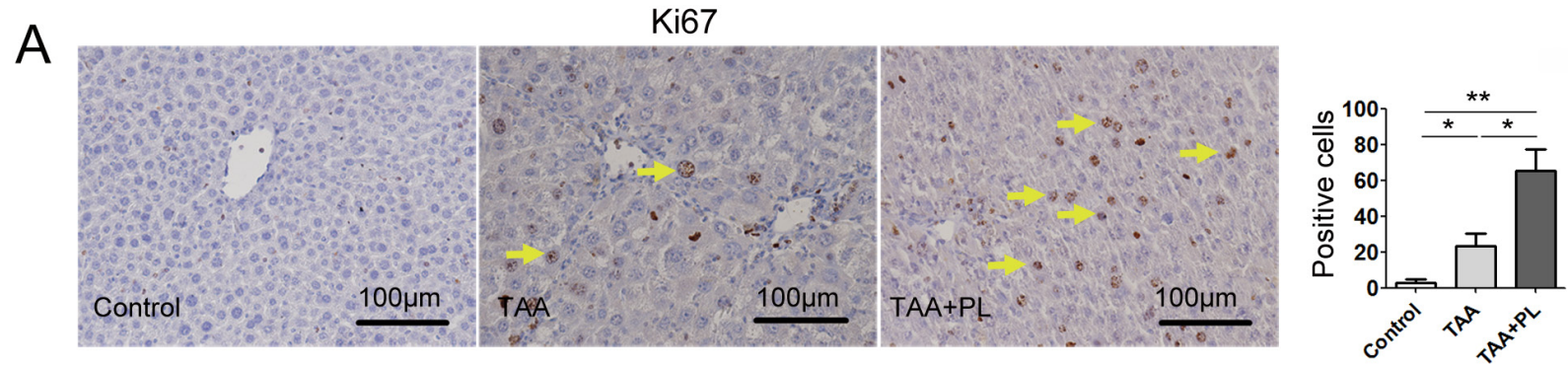

B

Cleaved caspase-3
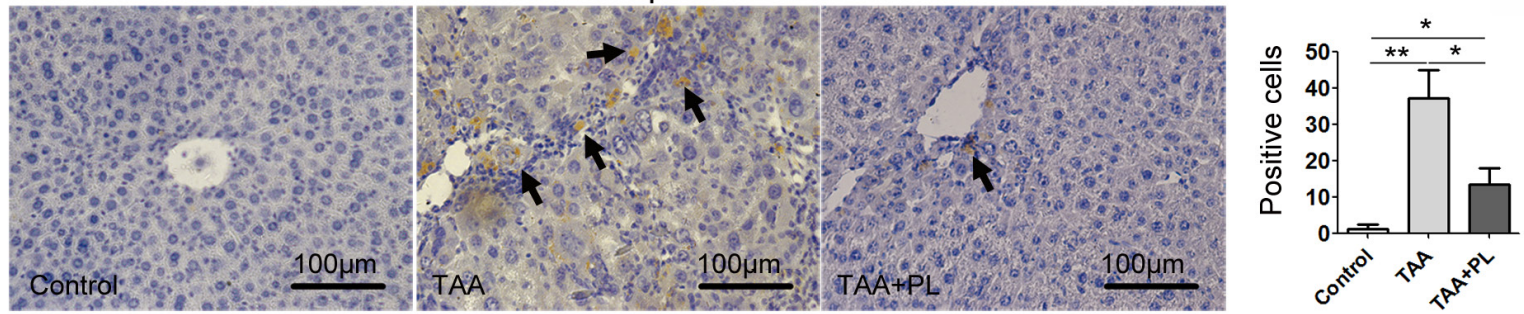

Figure 5: Plumbagin enhanced hepatocellular proliferation and inhibited hepatocellular apoptosis in a chronic liver fibrosis model. A. Ki67 (yellow arrows), a marker of proliferation. B. Cleaved caspase-3, a marker of apoptosis (black arrows). The sections were incubated with HRP-conjugated secondary antibodies and developed using DAB (brown). The sections were counterstained with hematoxylin (dark blue). Data are expressed as means $\pm \mathrm{SD}$, and data are representative of $4-6$ mice/group. ${ }^{*} \mathrm{p}<0.05 ;{ }^{* *} \mathrm{p}<0.01$. Statistical analyses were performed using Student's t tests.
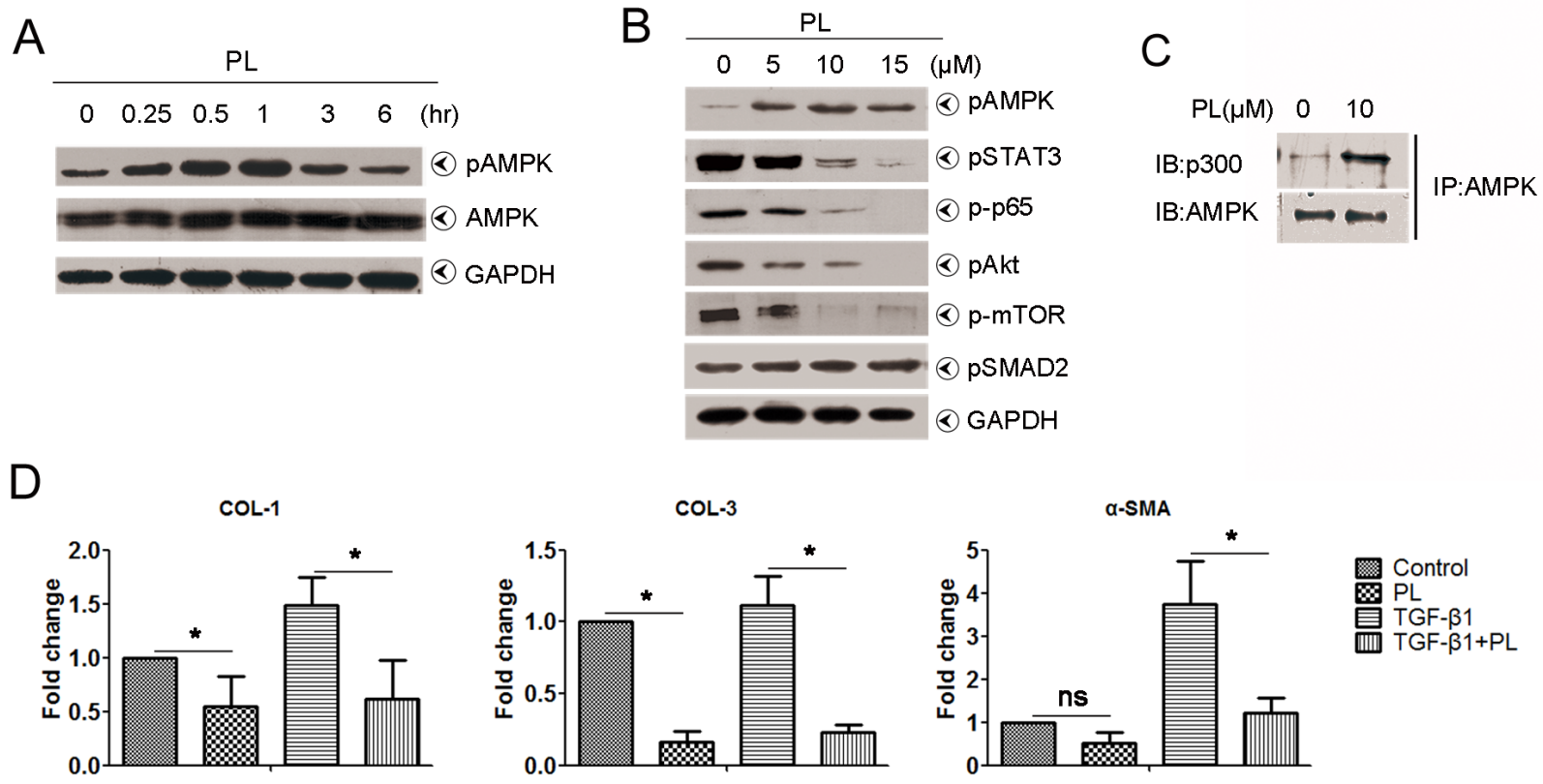

Figure 6: Effects of plumbagin on human hepatic stellate cells. A. Plumbagin promoted pAMPK levels in a time-dependent manner. LX-2 cells were treated with $10 \mu \mathrm{M}$ plumbagin for the indicated times, and pAMPK levels were analyzed. B. Plumbagin inhibited pSTAT3, p-p65, pAkt and p-mTOR levels in a dose-dependent manner. However, plumbagin had no effect on pSMAD2. LX-2 cells were first treated with the indicated concentrations of plumbagin for $1 \mathrm{~h}$ after incubation. Whole cells were harvested and analyzed using Western blots for pAMPK, pSTAT3, p-p65, pAkt, p-mTOR and pSMAD2. GAPDH was used as a loading control. C. Plumbagin facilitated the bind of AMPK with p300. LX-2 cells were treated with $10 \mu \mathrm{M}$ plumbagin for $1 \mathrm{~h}$, and cell lysates were immunoprecipitated using an antiAMPK antibody, followed by immunoblotting with an anti-p300 or anti-AMPK antibody. D. Plumbagin decreased mRNA level of fibrosisassociated genes. LX-2 cells were treated with plumbagin $(200 \mathrm{ng} / \mathrm{ml})$ or TGF- $\beta 1(2 \mathrm{ng} / \mathrm{ml})$ or combination for $24 \mathrm{~h}$, and then total RNA were extracted for quantitative PCR of COL-1, COL-3 and $\alpha$-SMA. Representative images are shown for all panels. Data are expressed as means \pm SD. ${ }^{*} \mathrm{p}<0.05 ; * * \mathrm{p}<0.01$. p values were calculated using a two-sided Student's t test. 
is consistent with previous studies [32, 33]. These results support the conclusion that plumbagin inhibits macrophage recruitment and suppresses NF- $\mathrm{kB}$ activation in macrophages.

Liver fibrosis results from the deposition of extracellular matrix proteins that are primarily produced by $\alpha$-SMA-positive HSCs/myofibroblasts $[14,17]$. The present study demonstrated that plumbagin treatment suppressed HSCs/myofibroblasts activation (measured as $\alpha$-SMA) in livers in vivo and the induction of LX-2 cell apoptosis in vitro. Plumbagin therapy remarkably diminished TAA-induced fibrogenesis, which was verified using picrosirius red staining, the Ishak fibrosis score and immunostaining for COL-1/3 in liver sections from mice in the present experimental model. TGF- $\beta 1$ signaling is tightly associated with the fibrotic response. In the present study, we found that plumbagin treatment reduced expression of TGF- $\beta 1$ in livers.

AMPK activation is relevant for the development of hepatic fibrosis due to its inactivation of HSCs. Activation of AMPK suppresses the activity of HSCs by inhibiting platelet-derived growth factor (PDGF)-induced mitogenesis and migration, in addition to downregulating monocyte chemoattractant protein-1 (MCP-1) protein secretion [19]. Activation of AMPK reduces HSC proliferation and sensitizes activated HSCs to apoptosis by modulating suppressors of cytokine signaling (SOCS-3) expression [34]. In our recent study, we found that activation of AMPK can dampen the activation and proliferation of hepatic stellate cells induced by TGF- $\beta 1[14,15]$. AMPK activation can interrupt SMAD complex association with p300 [18], which, as a SMAD transcriptional cofactor, is required by the induction of fibrosis gene expression by SMAD complex [20, 35]. In the present study, we showed the highly affinity of AMPK to p300 in the presence of plumbagin in vitro. Moreover, plumbagin decreased mRNA level of fibrosis-associated genes, such as COL1, COL3 and $\alpha$-SMA, which all were downstream gene of TGF- $\beta 1$ pathway.

$\mathrm{NF}-\kappa \mathrm{B}$ is a previously confirmed HSC survival factor [36]. Macrophages also contribute to liver fibrosis via the promotion of NF- $\mathrm{KB}$ activation in HSCs [21]. The Akt/mTOR pathway correlates HSC survival and proliferation [22-24]. Activated Akt is a key survival factor that directly phosphorylates mTOR, which ultimately stimulates HSC proliferation [25]. STAT3 actively promotes HSC activation [37, 38]. Plumbagin treatment inhibited all of the aforementioned signals that are associated with HSCs activation in LX-2 cells in vitro. These results support that plumbagin inhibited liver fibrogenesis by targeting HSCs.

In summary, the present study demonstrated that plumbagin can exert protective effects on liver injury. As summarized in Figure 7, plumbagin increased survival rate, reduced liver congestion and inflammation, and blocked the recruitment of macrophages in the FHF model. In addition, plumbagin treatment remarkably diminished liver fibrosis and inflammation in the chronic liver injury

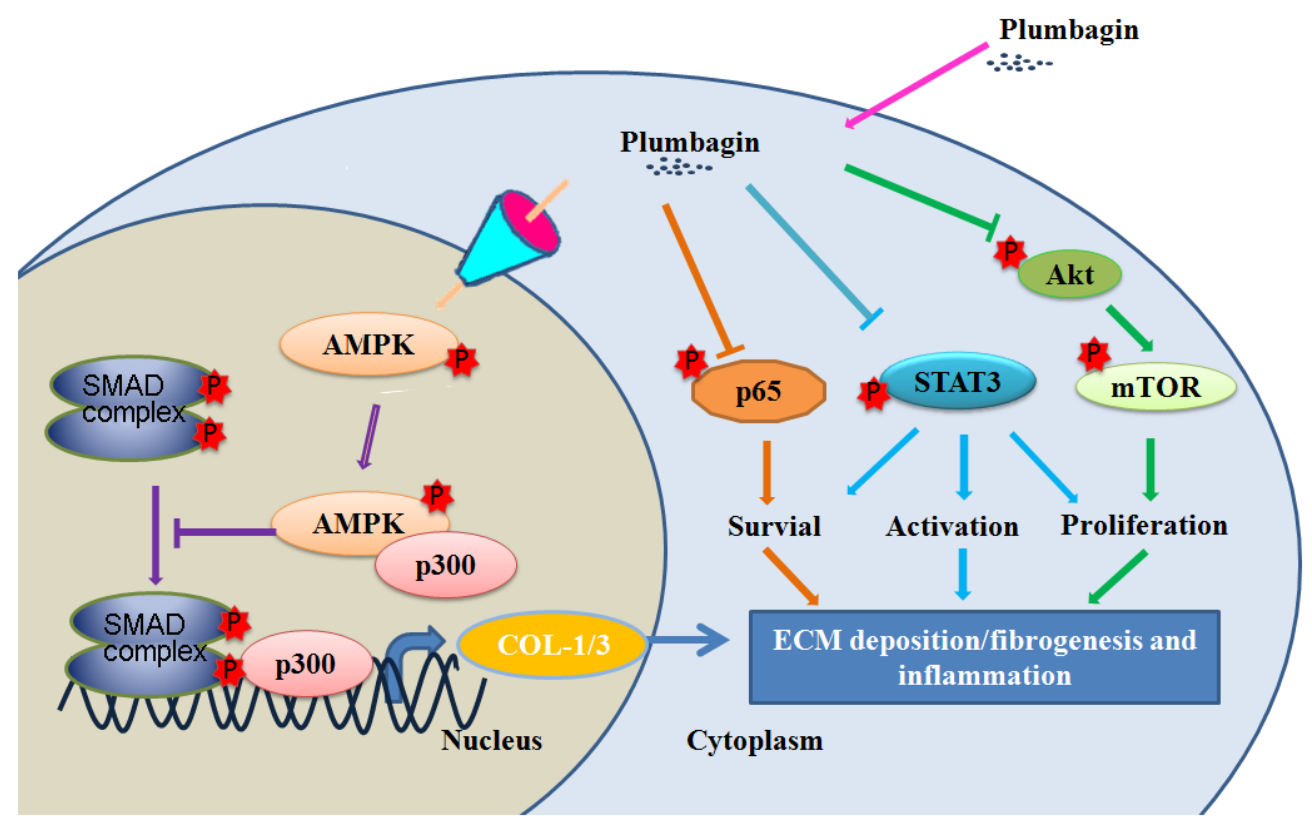

Figure 7: Liver protection mechanism of plumbagin. Plumbagin increased AMPK phosphorylation that promoted AMPK binding to $\mathrm{p} 300$, which is a SMAD transcriptional cofactor. This may further competitively decreases the p300/SMAD complex initiated transcription for COL-1/3 and $\alpha$-SMA. Plumbagin also attenuated NF- $\kappa$ B, STAT3, and Akt/mTOR signals in LX-2 cells, which were involved in proinflammation and survival of HSCs/myofibroblasts. In conclusion, these findings indicate that plumbagin may be a powerful drug candidate to protect the liver from acute and chronic damage by inhibiting inflammation and collagen production. 
model. Furthermore, plumbagin significantly suppress the HSC/myofibroblasts activation by reduced expression of markers $\alpha$-SMA and COL-1/3, and reduced F4/80 positive macrophage in liver. Plumbagin increased AMPK phosphorylation that promoted AMPK binding to $\mathrm{p} 300$, which is a SMAD transcriptional cofactor. This decreases the p300/SMAD complex initiated transcription for COL$1 / 3$ and $\alpha$-SMA. Moreover, plumbagin attenuated NF- $\kappa B$, STAT3, and Akt/mTOR signals in LX-2 cells, which were involved in pro-inflammation and survival of HSCs. In addition, NF- $\kappa \mathrm{B}$ signal also hampered with plumbagin. It is expected that plumbagin may be a powerful candidate to protect the liver from acute and chronic damage by inhibiting inflammation and collagen production.

\section{MATERIALS AND METHODS}

\section{Reagents and animals}

TAA, sirius red, and a 10\% formalin solution were purchased from Sigma Aldrich (St. Louis, MO, USA). Plumbagin (J\&K, Beijing, China) was prepared in dimethyl sulfoxide (DMSO) and diluted with PBS. ICR female mice were purchased from the Academy of Military Medical Science (Beijing, China) and weighed 20-22 g at the time of experiments. All mice were acclimatized for 1 week prior to the beginning of all experiments. Mice were housed in an animal room with a 12-hour light/ dark cycle. All experimental protocols were approved by Tianjin Medical University Animal Ethics Committee and all methods were carried out in accordance with the approved guidelines.

\section{Experimental model}

FHF model (diagrammed in Supplementary Figure 2A) was induced using a single intraperitoneal injection of TAA $(300 \mu \mathrm{g} / \mathrm{g}$ bw) $[14,15]$. Plumbagin $(2 \mu \mathrm{g} / \mathrm{g}$ bw) [16] was administered intragastrically to PL+TAA mice 2 days before TAA injection until the end of the experiment.

Chronic liver fibrosis model [14, 15] (diagrammed in Supplementary Figure 2B). TAA (100 $\mu \mathrm{g} / \mathrm{g}$ bw ip, 3 times weekly) was injected intraperitoneally into TAA+PL and TAA mice for the first two weeks, and the dose of TAA was increased to $200 \mu \mathrm{g} / \mathrm{g}$ until the sixth week. Plumbagin $(2 \mu \mathrm{g} / \mathrm{g} /$ day bw ig) was administered after 6 TAA administrations. Mice were randomized into 3 groups: (i) Control group; (ii) TAA+PL mice, which were treated with TAA and plumbagin; and (iii) TAA mice, which were treated with TAA only.

All mice were sacrificed at specific times after TAA administration.

\section{ALT and ALP}

Serum samples were collected using orbital venous plexus bleeding in anesthetized mice. Alanine transaminase (ALT) and alkaline phosphatase (ALP) activities were determined using an EnzyChromTM Alanine Transaminase Assay Kit (BioAssay Systems) and a QuantiChromTM Alkaline Phosphatase Assay Kit (BioAssay Systems) [14, 15], respectively.

\section{Histology}

Livers were perfused with PBS and removed. Images were obtained, and livers were weighed and cut into pieces. Liver specimens were fixed in a neutralbuffered formalin solution, embedded in paraffin, and cut into $7 \mu \mathrm{m}$ sections.

Liver slices were stained with hematoxylin-eosin (HE) to identify inflammation and necrosis, picrosirius red for fibrosis identification and Necroinflammation and fibrosis were scored using an Ishak system [14, 15, 39] (Details are described in Supplementary Table 1, 2). Liver slices were stained with periodic acid-Schiff (PAS) staining for analyses of glycogen metabolism. Positive staining was quantified using Image-Pro Plus 6 Windows Software (Media Cybernetics, USA).

\section{Immunostaining}

Tissue sections were incubated with the following primary antibodies: rabbit anti- $\alpha$-SMA (1:200; Abcam), anti-F4/80 (AbD Serotec, Oxford, UK), antimyeloperoxidase antibody (1:200; Abcam), rabbit anticollagen 1 (1:200; Abcam), anti-collagen 3 (1:1,000; Abcam), rabbit anti-TGF $\beta 1$ (1:100; Sigma-Aldrich), anti-Ki67 (Abcam, ab66155), and anti-cleaved caspase-3 (Asp 175) (Cell Signaling Technology, Inc., CAT\#: 9664). Sections for immunohistochemistry were incubated with horseradish peroxidase (HRP)-conjugated secondary antibodies (1:1000; Jackson, 11-GAR007, distributed by M $\mu$ ltiSciences Biotech Co., Ltd.) and developed using 3,3'-diaminobenzidine (DAB). Alexa 555-conjugated secondary antibodies (1:200; Molecular Probes) were used for immunofluorescence. Sections were counterstained with hematoxylin or DAPI (eBioscience, 00-4959). Positive staining was quantified by manual method or Image-Pro Plus 6 Windows Software (Media Cybernetics, USA).

\section{Hepatic MCP-1 assay}

Liver tissues were homogenized in RIPA (Applygen Technologies Inc, Beijing, China) by Bullet Blender Homogenizer (USA). Hepatic MCP-1 content was determined with Mouse monocyte chemotactic protein 1 ELISA Kit (Suobio, China). The procedures are according to manufacturers' protocols.

\section{Western blotting}

Total protein from LX-2 were extracted using RIPA Lysis Buffer (Beijing Biomed) and the protease 
inhibitor PMSF, and the nuclear proteins were extracted using the Nucleoprotein Extraction Kit (Sangon Biotech, Shanghai). Cell lysates were separated using SDS-PAGE and transferred to Immobilon-P membranes (Millipore). Membranes were incubated with primary antibodies for AMPK (Cell Signaling Technology), pAMPK (Cell Signaling Technology), pSTAT3 (Cell Signaling Technology), pAkt (Cell Signaling Technology), p-mTOR (Cell Signaling Technology), p-SMAD2 (Sigma-Aldrich), and GAPDH (Santa Cruz Biotechnology) followed by HRP-conjugated secondary antibodies (1:2,000; Sungene Biotech Co., Ltd) that were specific to the species of the primary antibodies.

\section{Immunoprecipitation}

The primary antibodies were AMPK and p300 (Cell Signaling Technology), and HRP-conjugated secondary antibodies (1:2,000; Sungene Biotech Co., Ltd.) that were specific to the species of the primary antibodies were used. Immunoprecipitation was performed in accordance with the protocol of Dynabeads ${ }^{\circledR}$ Protein G (Life Technologies AS, Norway).

\section{Gene expression}

LX-2 cells were treated with plumbagin $(200 \mathrm{ng} / \mathrm{ml})$ or TGF- $\beta 1(2 \mathrm{ng} / \mathrm{ml})$ or combination for $24 \mathrm{~h}$, and then total RNA was extracted by using Trizol reagent according to the manufacturer's instructions (Invitrogen, Carlsbad, USA). The RNA was converted into cDNA with random hexamers and M-MLV reverse transcriptase (Invitrogen, Oregon, USA). PCR was performed for COL-1/3, $\alpha$-SMA and GAPDH detection. Primers were from BGI, China (Supplementary Table 3).

\section{Statistical analysis}

Data are expressed as means \pm SD (standard deviation). Statistical comparisons between experimental groups were performed using Student's t-test.

\section{ACKNOWLEDGMENTS}

This work is supported by the Ministry of Science and Technology of China through Grant No. 2012CB932503; the National Natural Science Foundation of China through Grant 81172864, 81272317, 81270891, 81302568 and 81301026; Shandong College Outstanding Young Teacher Domestic Visiting Scholar Project.

\section{CONFLICTS OF INTEREST}

Competing financial interests: The authors declare no competing financial interests.

\section{Author contributions}

H. Z. and R. Z. conception and design of research; H. Z., X. C., Y. Z., Z. G., G. M. and D. W. performed experiments; H. Z., and H. W. analyzed data; H. Z., F. Y., Q. Z., Z. X., D. L., Y. L., L. Z. and H. W. interpreted results of experiments; H. Z., Y. D. and H. W. prepared figures; H. Z., and H. W. drafted manuscript; F. G., Z. Y., L. Q., L. K. and R. Z. edited and revised manuscript; R. Z. approved final version of manuscript.

\section{REFERENCES}

1. Wallace K, Burt A, Wright M. Liver fibrosis. Biochem J. 2008; 411:1-18.

2. Bernal W, Auzinger G, Dhawan A, Wendon J. Acute liver failure. The Lancet. 2010; 376:190-201.

3. Wallace K, Burt AD, Wright MC. Liver fibrosis. Biochemical Journal. 2008; 411:1-18.

4. Parimala R, Sachdanandam P. Effect of Plumbagin on some glucose metabolising enzymes studied in rats in experimental hepatoma. Molecular and cellular biochemistry. 1993; 125:59-63.

5. Sharma I, Gusain D, Dixit VP. Hypolipidaemic and antiatherosclerotic effects of plumbagin in rabbits. Indian journal of physiology and pharmacology. 1991; 35:10-14.

6. Parimala R, Sachdanandam P. Effect of Plumbagin on some glucose metabolising enzymes studied in rats in experimental hepatoma. Mol Cell Biochem. 1993; 125:59-63.

7. Sugie S, Okamoto K, Rahman KM, Tanaka T, Kawai K, Yamahara J, Mori H. Inhibitory effects of plumbagin and juglone on azoxymethane-induced intestinal carcinogenesis in rats. Cancer letters. 1998; 127:177-183.

8. Shih YW, Lee YC, Wu PF, Lee YB, Chiang TA. Plumbagin inhibits invasion and migration of liver cancer HepG2 cells by decreasing productions of matrix metalloproteinase-2 and urokinase- plasminogen activator. Hepatology research. 2009; 39:998-1009.

9. Xue Z, Ge Z, Zhang K, Sun R, Yang J, Han R, Peng M, Li Y, Li W, Zhang D, Hao J, Da Y, Yao Z, Zhang R. Embelin suppresses dendritic cell functions and limits autoimmune encephalomyelitis through the TGF-beta/beta-catenin and STAT3 signaling pathways. Molecular neurobiology. 2014; 49:1087-1101.

10. Ge Z, Da Y, Xue Z, Zhang K, Zhuang H, Peng M, Li Y, Li W, Simard A, Hao J, Yao Z, Zhang R. Vorinostat, a histone deacetylase inhibitor, suppresses dendritic cell function and ameliorates experimental autoimmune encephalomyelitis. Experimental neurology. 2013; 241:56-66.

11. Li W, Zhang Z, Zhang K, Xue Z, Li Y, Zhang Z, Zhang L, Gu C, Zhang Q, Hao J, Da Y, Yao Z, Kong Y, Zhang R. Arctigenin Suppress Th17 Cells and Ameliorates 
Experimental Autoimmune Encephalomyelitis Through AMPK, PPAR-gamma/ROR-gammat Signaling. Molecular neurobiology. 2016; 53:5356-5366.

12. Zhang K, Ge Z, Xue Z, Huang W, Mei M, Zhang Q, Li Y, Li W, Zhang Z, Zhang Z. Chrysin suppresses human CD14+ monocyte-derived dendritic cells and ameliorates experimental autoimmune encephalomyelitis. Journal of neuroimmunology. 2015; 288:13-20.

13. Zhang K, Ge Z, Da Y, Wang D, Liu Y, Xue Z, Li Y, Li W, Zhang L, Wang H, Zhang H, Peng M, Hao J, Yao Z, Zhang R. Plumbagin suppresses dendritic cell functions and alleviates experimental autoimmune encephalomyelitis. Journal of neuroimmunology. 2014; 273:42-52.

14. Wang H, Zhang H, Zhang Z, Huang B, Cheng X, Wang D, la Gahu Z, Xue Z, Da Y, Li D. Adiponectin-derived active peptide ADP355 exerts anti-inflammatory and anti-fibrotic activities in thioacetamide-induced liver injury. Scientific reports. 2016; 6:19445.

15. Huang B, Cheng X, Wang H, Huang W, la Ga Hu Z, Wang D, Zhang K, Zhang H, Xue Z, Da Y, Zhang N, Hu Y, Yao Z, Qiao L, Gao F, Zhang R. Mesenchymal stem cells and their secreted molecules predominantly ameliorate fulminant hepatic failure and chronic liver fibrosis in mice respectively. J Transl Med. 2016; 14:45.

16. Sung B, Oyajobi B, Aggarwal BB. Plumbagin inhibits osteoclastogenesis and reduces human breast cancerinduced osteolytic bone metastasis in mice through suppression of RANKL signaling. Molecular cancer therapeutics. 2012; 11:350-359.

17. Bedossa P, Paradis V. Liver extracellular matrix in health and disease. J Pathol. 2003; 200:504-515.

18. Leask A, Abraham DJ. TGF-beta signaling and the fibrotic response. FASEB journal. 2004; 18:816-827.

19. Caligiuri A, Bertolani C, Guerra CT, Aleffi S, Galastri S, Trappoliere M, Vizzutti F, Gelmini S, Laffi G, Pinzani M. Adenosine monophosphate-activated protein kinase modulates the activated phenotype of hepatic stellate cells. Hepatology. 2008; 47:668-676.

20. Lim JY, Oh MA, Kim WH, Sohn HY, Park SI. AMPactivated protein kinase inhibits TGF-beta-induced fibrogenic responses of hepatic stellate cells by targeting transcriptional coactivator p300. Journal of cellular physiology. 2012; 227:1081-1089.

21. Pradere JP, Kluwe J, Minicis S, Jiao JJ, Gwak GY, Dapito DH, Jang MK, Guenther ND, Mederacke I, Friedman R. Hepatic macrophages but not dendritic cells contribute to liver fibrosis by promoting the survival of activated hepatic stellate cells in mice. Hepatology. 2013; 58:1461-1473.

22. Reif S, Lang A, Lindquist JN, Yata Y, Gabele E, Scanga A, Brenner DA, Rippe RA. The role of focal adhesion kinasephosphatidylinositol 3-kinase-akt signaling in hepatic stellate cell proliferation and type I collagen expression. The Journal of biological chemistry. 2003; 278:8083-8090.

23. Foo NP, Lin SH, Lee YH, Wu MJ, Wang YJ. alpha-Lipoic acid inhibits liver fibrosis through the attenuation of ROStriggered signaling in hepatic stellate cells activated by PDGF, TGF-beta. Toxicology. 2011; 282:39-46.

24. Saxena NK, Titus MA, Ding X, Floyd J, Srinivasan S, Sitaraman SV, Anania FA. Leptin as a novel profibrogenic cytokine in hepatic stellate cells: mitogenesis and inhibition of apoptosis mediated by extracellular regulated kinase (Erk) and Akt phosphorylation. The FASEB journal. 2004; 18:1612-1614.

25. Liu Q, Chen J, Wang X, Yu L, Hu LH, Shen X. Withagulatin A inhibits hepatic stellate cell viability and procollagen I production through Akt and Smad signaling pathways. Acta pharmacologica Sinica. 2010; 31:944-952.

26. Wynn TA, Barron L. Macrophages: master regulators of inflammation and fibrosis. Seminars in liver disease. 2010; 30:245-257.

27. Duffield JS, Forbes SJ, Constandinou CM, Clay S, Partolina M, Vuthoori S, Wu S, Lang R, Iredale JP. Selective depletion of macrophages reveals distinct, opposing roles during liver injury and repair. Journal of Clinical Investigation. 2005; 115:56-65.

28. Leibovich S, Ross R. The role of the macrophage in wound repair. A study with hydrocortisone and antimacrophage serum. The American journal of pathology. 1975; 78:71.

29. Phillipson M, Kubes P. The neutrophil in vascular inflammation. Nature medicine. 2011; 17:1381-1390.

30. Jaeschke H, Hasegawa T. Role of neutrophils in acute inflammatory liver injury. Liver International. 2006; 26:912-919.

31. Mandrekar P, Szabo G. Signalling pathways in alcoholinduced liver inflammation. J Hepatol. 2009; 50:1258-1266.

32. Wang T, Wu F, Jin Z, Zhai Z, Wang Y, Tu B, Yan W, Tang T. Plumbagin inhibits LPS-induced inflammation through the inactivation of the nuclear factor-kappa B and mitogen activated protein kinase signaling pathways in RAW 264.7 cells. Food and Chemical Toxicology. 2014; 64:177-183.

33. Checker R, Patwardhan RS, Sharma D, Menon J, Thoh M, Sandur SK, Sainis KB, Poduval TB. Plumbagin, a vitamin K3 analogue, abrogates lipopolysaccharide-induced oxidative stress, inflammation and endotoxic shock via NF-kappaB suppression. Inflammation. 2014; 37:542-554.

34. Handy JA, Saxena NK, Fu P, Lin S, Mells JE, Gupta NA, Anania FA. Adiponectin activation of AMPK disrupts leptin-mediated hepatic fibrosis via suppressors of cytokine signaling (SOCS-3). Journal of cellular biochemistry. 2010; 110:1195-1207.

35. Park SR, Jung MH, Jeon SH, Park MH, Park KH, Lee MR, Kim PH. IFN-gamma down-regulates TGF-beta1-induced 
IgA expression through Stat1 and p300 signaling. Molecules and cells. 2010; 29:57-62.

36. Oakley F, Meso M, Iredale JP, Green K, Marek CJ, Zhou X, May MJ, Millward-Sadler H, Wright MC, Mann DA. Inhibition of inhibitor of $\kappa \mathrm{B}$ kinases stimulates hepatic stellate cell apoptosis and accelerated recovery from rat liver fibrosis. Gastroenterology. 2005; 128:108-120.

37. Meng F, Wang K, Aoyama T, Grivennikov SI, Paik Y, Scholten D, Cong M, Iwaisako K, Liu X, Zhang M. Interleukin-17 signaling in inflammatory, Kupffer cells, and hepatic stellate cells exacerbates liver fibrosis in mice. Gastroenterology. 2012; 143:765-776. e763.

38. Wang H, Lafdil F, Kong X, Gao B. Signal transducer and activator of transcription 3 in liver diseases: a novel therapeutic target. International journal of biological sciences. 2011; 7:536.

39. Ishak K, Baptista A, Bianchi L, Callea F, De Groote J, Gudat F, Denk H, Desmet V, Korb G, MacSween RN. Histological grading and staging of chronic hepatitis. J hepatol. 1995; 22:696-699. 